\title{
Metabolic Syndrome and Cancer
}

\author{
Pooja Pothiwala, M.D., ${ }^{1}$ Sushil K. Jain, Ph.D., ${ }^{2}$ and Subhashini Yaturu, M.D. ${ }^{3}$
}

\begin{abstract}
Since its first description by Reavan in 1988, accepted criteria for clinical identification of the components of metabolic syndrome have been promulgated by the National Cholesterol Education Program/Adult Treatment Panel III (NCEP/ATP III) and the World Health Organization (WHO) as well as the International Diabetes Federation (IDF), and the American Association of Clinical Endocrinologists (AACE). Insulin resistance is a common metabolic abnormality underlying type 2 diabetes mellitus and is also an independent risk factor for cardiovascular disease. Although ATP III identified cardiovascular disease (CVD) as the primary clinical outcome of the metabolic syndrome, we now have evidence that metabolic syndrome is associated with type 2 diabetes mellitus, polycystic ovarian disease, nonalcoholic fatty liver disease, and possibly some cancers. This review summarizes evidence in support of the relationship between metabolic syndrome and various cancers and possible underlying mechanisms and therapeutic interventions.
\end{abstract}

\section{Introduction}

Since its first Description by Reavan in $1988,{ }^{1}$ accepted Criteria for clinical identification of the components of metabolic syndrome have been promulgated by the National Cholesterol Education Program/Adult Treatment Panel III (NCEP-ATP III) ${ }^{2}$ and the World Health Organization (WHO) as well as the International Diabetes Federation (IDF), ${ }^{3}$ and the American Association of Clinical Endocrinologists (AACE). ${ }^{4}$ Insulin resistance is a common metabolic abnormality underlying type 2 diabetes mellitus and is also an independent risk factor for cardiovascular disease. ${ }^{5,6}$ Although ATP III identified cardiovascular disease (CVD) as the primary clinical outcome of the metabolic syndrome, we now have evidence that metabolic syndrome is associated with type 2 diabetes mellitus, polycystic ovarian disease, nonalcoholic fatty liver disease (NAFLD), and possibly some cancers. This review summarizes evidence in support of the relationship between metabolic syndrome and various cancers and possible underlying mechanisms and therapeutic interventions.

\section{Definition of Metabolic Syndrome}

Various definitions developed by different organizations have been used to define metabolic syndrome. Traditionally, it has been defined by WHO, NCEP/ATP III, and IDF by the criteria shown in Table 1 . Recently, a consensus statement from the IDF has provided more race-specific criteria in defining waist circumference (WC), thus modifying this criterion in such a way to make it more useful in non-Caucasian populations (Table 1$)^{3}$

\section{Metabolic Syndrome and Cancer}

Analysis of data on 8814 men and women aged 20 years or older from the Third National Health and Nutrition Examination Survey (NHANES) showed that about 47 million U.S. residents have the metabolic syndrome, with the highest prevalence of $43.5 \%$ found in people over age $60 .{ }^{8}$ In the last several years, several interesting studies have been published showing an association between cancer risk and the different components of metabolic syndrome. However, epidemiologic studies linking metabolic syndrome to cancer are scarce. Recently, a large population-based study was done in Milan, Italy, that enrolled 16,677 subjects who were on medications for hyperlipidemia, diabetes and hypertension and followed them for up to 8 years. A total of 823 incidents of cancer occurred during the study period, including a significantly increased risk of pancreatic cancer in males (standardized incidence ratio 178 [114-266]) and colorectal cancer in females (standardized incidence ratio 133 [101-170]) were observed. Nonsignificantly increased risks were also observed in women for liver, gallbladder and biliary tract, breast, and endometrial cancers. ${ }^{9}$

${ }^{1}$ Department of Endocrinology and Metabolism, Overton Brooks Veterans Affairs Medical Center, Shreveport, Louisiana.

${ }^{2}$ Department of Pediatrics and ${ }^{3}$ Department of Endocrinology, Louisiana State University Health Sciences Center, Shreveport, Louisiana. 
Table 1. Various Definitions of Metabolic Syndrome

\begin{tabular}{|c|c|c|c|}
\hline Risk factors & $\begin{array}{l}\text { NCEP/ATP III } \\
\text { (3 of } 5 \text { criteria } \\
\text { necessary })^{2}\end{array}$ & $\begin{array}{l}\text { WHO } 1999 \text { (impaired glucose } \\
\text { regulation or hyperinsulinemia } \\
\text { and } 2 \text { or more criteria necessary) }\end{array}$ & $\begin{array}{l}\text { IDF (increased waist } \\
\text { circumference plus } \\
\text { any } 2 \text { of other } 4 \\
\text { criteria) }^{99}\end{array}$ \\
\hline Fasting glucose & $\geq 110 \mathrm{mg} / \mathrm{dL}$ & $\geq 110 \mathrm{mg} / \mathrm{dL}$ & $\geq 110 \mathrm{mg} / \mathrm{dL}$ \\
\hline Prandial glucose & & $>140 \mathrm{mg} / \mathrm{dL}$ & \\
\hline Hyperinsulinemia & & $\begin{array}{l}\text { Fasting serum insulin: third } \\
\text { quartile for control group }\end{array}$ & \\
\hline Hypertriglyceridemia $^{\mathrm{b}}$ & $\geq 150 \mathrm{mg} / \mathrm{dL}$ & $\geq 150 \mathrm{mg} / \mathrm{dL}$ & $\geq 150 \mathrm{mg} / \mathrm{dL}$ \\
\hline \multicolumn{4}{|l|}{ Low HDL } \\
\hline Men & $<40 \mathrm{mg} / \mathrm{dL}$ & $<35 \mathrm{mg} / \mathrm{dL}$ & $<40 \mathrm{mg} / \mathrm{dL}$ \\
\hline Women & $<50 \mathrm{mg} / \mathrm{dL}$ & $<39 \mathrm{mg} / \mathrm{dL}$ & $<50 \mathrm{mg} / \mathrm{dL}$ \\
\hline Abdominal obesity & & waist: hip ratio & \\
\hline Men & $>102 \mathrm{~cm}$ & $>0.9$ in & $\geq 94 \mathrm{~cm}$ \\
\hline Women & $>88 \mathrm{~cm}$ & $>0.85$ in & $\geq 80 \mathrm{~cm}$ \\
\hline Hypertension $^{\mathrm{b}}$ & $\geq 130 / \geq 85 \mathrm{~mm} \mathrm{Hg}$ & $\geq 140 / \geq 90 \mathrm{~mm} \mathrm{Hg}$ & $\geq 130 / \geq 85 \mathrm{~mm}$ \\
\hline Microalbuminuria & & $\geq 20 \mu \mathrm{g} / \mathrm{min}$ & \\
\hline
\end{tabular}

aWorld Health Organization. Definition, diagnosis and classification of diabetes mellitus and its complications: report of a WHO consultation. Part 1: diagnosis and classification of diabetes mellitus. Geneva, Switzerland: World Health Organization, 1999.

${ }^{b}$ Considered positive criteria if person is on medications for lipids, hypertension, or hyperglycemia regardless of value.

Abbreviations: NCEP/ATP III, National Cholesterol Education Program/Adult Treatment Panel III; WHO, World Health Organization; IDF, International Diabetes Federation; HDL, high-density lipoprotein.

\section{Obesity and Cancer}

Obesity, which is a component of metabolic syndrome, has been associated with high risks of cancers at the multiple sites. Accumulating epidemiologic evidence shows that excess body weight is associated with an increased risk of several common adult cancers. An association between obesity or an incremental increase in body mass index (BMI) and an increased cancer risk have been reported for colon cancer (men and women) and rectal cancer (men only), ${ }^{10}$ liver cancer, ${ }^{11}$ multiple myeloma, ${ }^{12}$ non-Hodgkin's lymphoma, ${ }^{13}$ gallbladder ${ }^{14}$ pancreatic cancer ${ }^{15}$ leukemia, ${ }^{16}$ ovarian, ${ }^{17}$ breast cancer, ${ }^{18}$ and endometrial cancer. ${ }^{19,20}$ A recent study from the United Kingdom showed that increasing BMI was associated with an increased incidence of endometrial cancer (trend in relative risk per 10 units $=2.89,95 \%$ confidence interval $[\mathrm{CI}]$ 2.62-3.18), adenocarcinoma of the esophagus (2.38, 1.59-3.56), kidney cancer (1.53, 1.27-1.84), leukemia (1.50, 1.23-1.83), multiple myeloma (1.31, 1.04-1.65), pancreatic cancer (1.24, 1.03-1.48), non-Hodgkin's lymphoma (1.17, 1.03-1.34), ovarian cancer (1.14, 1.03-1.27), all cancers combined (1.12, 1.09 -1.14), breast cancer in postmenopausal women (1.40, 1.31-1.49), and colorectal cancer in premenopausal women $(1.61,1.05-2.48){ }^{21}$ The same study also examined the status of menopause and BMI on different cancers in women, with an increased risk of colorectal cancer and malignant melanoma in premenopausal women and an increased risk for breast and endometrial cancers in postmenopausal women. ${ }^{21}$ Although the molecular mechanisms and the pathophysiology responsible are not fully understood, the most likely important mechanism seems to be insulin resistance. Chronic elevation of insulin is associated with the increased availability of insulin-like growth factor-1 (IGF-I). Other possible mechanisms include increased oxidative stress associated with obesity, which is especially relevant to obesity-related renal cancer. ${ }^{22}$
Recently, the same authors have implicated lipid peroxidation in the anticarcinogenic effect of suspected protective factors in breast cancer, such as soy, marine n-3 fatty acids, green tea, isothiocyanates, and vitamin D and calcium. ${ }^{23}$ Recent studies in the literature also have focused on excess adiposity and cancer outcome.

It is not surprising to note that increased adiposity may have a negative effect on treatment outcome and ultimate survival, because obesity has been found to be a negative prognostic factor for breast cancer ${ }^{24}$ and colon cancer. ${ }^{25,26}$

\section{Hyperglycemia and Cancer}

Hyperglycemia has also been linked to cancers in recent years independent of BMI. In 1990, the relationship between postload plasma glucose (after 50 grams of oral glucose) and 12-year cancer mortality was studied in 11,521 white men and 8591 white women aged 35-64 years upon enrollment in the Chicago Heart Association Detection Project (an industrysponsored study). Hyperglycemia was related to cancer mortality in men, but not in women, after controlling for age, BMI, smoking, serum cholesterol, systolic blood pressure, education, and antihypertensive treatment. ${ }^{27}$ Since then, various studies have confirmed these findings both in men and women. ${ }^{28,29}$ Recently, a prospective study in Sweden involving 74,207 men and women showed a modest reduction in prostate cancer risk in conjunction with diabetes and no significant association between hyperglycemia and cancer risk in men. However, after exclusion of prostate cancer (which accounted for two thirds of all male cancers), the data became weakly positive for an association with cancers in men. In women, hyperglycemia was associated significantly with cancer risk. There was also a significant increased risk of pancreatic cancer and malignant melanoma in both sexes and an increase risk of endometrial and breast cancer in women. ${ }^{30}$ 
Epidemiological studies suggest that men with type 2 diabetes are less likely than nondiabetic men to develop prostate cancer. Recent genetic studies have highlighted a potential genetic link between the two diseases. Two studies have identified a version (allele) of a variant in the HNF1B (also known as TCF2) gene that predisposes people to type 2 diabetes, and one of them showed that the same allele protects men from prostate cancer. ${ }^{31}$ Colorectal carcinoma and type 2 diabetes mellitus share common risk factors. and type 2 diabetes mellitus is associated with an increased risk of colorectal cancer. The increased risk occurs in both sexes. ${ }^{32,33}$ The hyperinsulinemia hypothesis is based on the premise that elevated plasma levels of insulin and free IGF-1 promote the proliferation of colon cells and confer a survival benefit upon transformed colon carcinoma cells. Chronic insulin therapy was associated with increased colorectal adenoma risk among type 2 diabetes mellitus patients. ${ }^{34,35}$

\section{Dyslipidemia and Cancer}

Dyslipidemia along with metabolic syndrome has been associated with multiple cancer risks. A recent prospective study from the United States examined the association between baseline plasma high-density lipoprotein cholesterol (HDL-C) levels and the incidence of lung cancer in 14,547 members of the Atherosclerosis Risk in Communities (ARIC) cohort. The hazard ratio of lung cancer incidence in relation to low HDL-C, adjusted for race, gender, exercise, alcohol consumption, BMI, triglycerides (TG), age, and cigarette pack-years of smoking, was 1.45 (95\% CI 1.10, 1.92). ${ }^{36}$ Extremely low levels of HDL ( $\leq 20 \mathrm{mg} / \mathrm{dL}$ ) are associated with an increased risk of malignancy with an odds ratio of 6.68 (95\% CI 1.8-24.5, $P=0.004) .{ }^{37}$ Low serum low-density lipoprotein cholesterol (LDL-C) levels ( $\leq 70 \mathrm{mg} / \mathrm{dL}$ ) are associated with increased odds of developing hematological cancer by more than 15-fold (OR 15.7, 95\% CI 1.78-138.4, $P=0.01) .{ }^{38}$ High TG have been shown to be associated with increased risk of postmenopausal breast and prostate cancer in multiple studies. ${ }^{39,40}$ Other components of metabolic syndrome, such as hypertension and elevated WC, have been also been linked modestly with cancer risk.

The following sections will discuss the available epidemiological data related to individual cancers as below.

\section{Colorectal cancer}

Epidemiologic data strongly support the observation that people with the metabolic syndrome are at increased risk of colon cancer and adenoma. ${ }^{41-47}$ Most of the studies do not clarify which component or components of the metabolic syndrome appear to have an additive effect on the development of colon cancer. ${ }^{44}$ In a Korean study, of all the individual metabolic syndrome components, abdominal obesity independently increased the risk for colonic precancerous lesions. ${ }^{46}$ A large study involving 368,277 participants found that among men, weight and BMI were associated with a higher risk of colon cancer (weight $\geq 90.0 \mathrm{~kg}$ vs. $<71 \mathrm{~kg}$, relative risk $[R R]=1.43,95 \% \mathrm{CI}=1.02-2.02 ; \mathrm{BMI} \geq 29.4 \mathrm{~kg} / \mathrm{m}^{2} \mathrm{vs}$. $\left.<23.6 \mathrm{~kg} / \mathrm{m}^{2}, \mathrm{RR}=1.55,95 \% \mathrm{CI}=1.12-2.15\right)$, but not among women. In both sexes, WC was positively related to risk of colon cancer (for men $\geq 103.0 \mathrm{~cm}$ vs. $<86.0 \mathrm{~cm}$ ], $\mathrm{RR}=1.39,95 \%$ $\mathrm{CI}=1.01-1.93$; for women $[\geq 89.0 \mathrm{~cm}$ vs. $<70.2 \mathrm{~cm}], \mathrm{RR}=1.48$, $95 \% \mathrm{CI}=1.08-2.03){ }^{48}$
Data from Physicians' Health Study (22,046 male physicians) showed that after adjusting for the increased prevalence of metabolic abnormalities with increasing age as well as after additional adjustment for all variables, the hazard ratios (HR) for two metabolic abnormalities and three or four metabolic abnormalities compared with no metabolic abnormality were both 1.4. For each metabolic abnormality separately, a BMI of $\geq 27 \mathrm{~kg} / \mathrm{m}^{2}$ and diabetes were independently associated with an increased risk for colorectal cancer with HRs of 1.4 (95\% CI, 1.1-1.7) and 1.5 (95\% CI, 1.1-2.0), respectively. Elevated blood pressure was associated with an only slight increase in risk (HR, 1.1; 95\% CI, 0.9-1.3), and hypercholesterolemia was associated with a slightly decreased risk for colorectal cancer with a HR of 0.9 (95\% CI, 0.7-1.1). ${ }^{49}$ Any such association in women is much weaker and controversial. Data analysis from nurse's health study showed after adjustment for age, that a history of diabetes was significantly associated with increased risk of colorectal cancer. The age-adjusted RR was $1.53(95 \% \mathrm{CI}=1.18-1.99 ; P=0.001) .{ }^{50}$ Another study involving postmenopausal women in the Iowa Women's Health Study $(n=35,230)$ showed that after adjusting for age, BMI, and other potential confounding variables, the RR for women with diabetes mellitus (DM) versus women without DM was modestly increased at 1.4 (95\% CI, 1.1-1.8). By anatomic subsite, the RR for proximal colon cancer was statistically significantly increased (RR, 1.9; 95\% CI, 1.3-2.6), whereas the RRs for distal colon (RR, 1.1; 95\% CI, $0.6-1.8)$ and rectal cancer (RR, 0.8; 95\% CI, 0.4-1.6) were not statistically different from unity. ${ }^{51}$ Thus, most of the data in women show some association with hyperglycemia but not BMI. It appears that waist circumference (WC) in women is more related to increased incidence of colon cancer than $\mathrm{BMI}$ is. One possible explanation is higher estrogen levels in obese women and use of hormone replacement therapy (HRT) as a confounding variable affecting the results.

Although the evidence is not yet conclusive, epidemiologic and animal evidence tends to support more strongly a direct role of hyperinsulinemia, as compared with other aspects of the insulin resistance syndrome for colon cancer risk. ${ }^{52}$ The mechanism underlying this association with hyperinsulinemia remains unknown; however, it may involve the influence of hyperinsulinemia in enhancing free or bioavailable insulin-like growth factor-1 (IGF-1) concentrations, which have been related to cancer risk. ${ }^{53}$

\section{Prostate cancer}

The association between prostate cancer and BMI is controversial. In a large retrospective study, the magnitude of increased risk in relation to BMI was modest; the RR of obese men (BMI $\geq 30)$ compared with those of normal weight was 1.09 (95\% CI, 1.04-1.15). However, the RR at age 50-59 years was 1.58 (95\% CI, 1.29-1.94) in men who became obese around age 45 years compared with those of normal weight. ${ }^{54}$ In a prospective study, Wolk et al. noted a positive relationship in cases diagnosed before age 60 and a negative relationship after age $80 .{ }^{55} \mathrm{~A}$ meta-analysis from Australlia that included 31 cohort studies and 25 case-control studies showed that overall RR for BMI was 1.05 per $5 \mathrm{~kg} / \mathrm{m}^{2}$ increment (95\% CI, 1.01-1.08). For studies that reported results by stage of disease, the RRs were greater for advanced disease (RR 1.12 per $5 \mathrm{~kg} / \mathrm{m}^{2}$ increment; 95\% CI, 1.01-1.23) compared with localized disease (RR 0.96 per $5 \mathrm{~kg} / \mathrm{m}^{2}$ increment; $95 \%$ 
CI, 0.89-1.03), $P=0.02$. Height was also positively associated with risk. ${ }^{56}$ In contradiction to the above data, the findings from the population-based case-control study reported by Robinson et all showed that obesity (BMI $\geq 30 \mathrm{~kg} / \mathrm{m}^{2}$ ) tended to be inversely associated with prostate cancer (odds ratio $[\mathrm{OR}]=0.79 ; 95 \% \mathrm{CI}, 0.46)$. The decreased risk was more pronounced at ages 20-29 years (OR $=0.53 ; 95 \% \mathrm{CI}, 0.28)$. Although these data have not been supported by other trials, the possible mechanisms may be changes in the free testosterone levels in early adulthood and associated changes in prostate morphology. ${ }^{57}$ Data from Cancer Prevention Study II Nutrition Cohort followed between 1992 and 2003 showed that the association between BMI in 1992 and risk of prostate cancer differed by stage and grade at diagnosis. BMI was inversely associated with risk of nonmetastatic low-grade prostate cancer (RR, 0.84; 95\% CI, 0.66-1.06), but positively associated with risk of nonmetastatic, high-grade prostate cancer (RR, 1.22; 95\% CI, 0.96-1.55) and risk of metastatic or fatal prostate cancer (RR, 1.54; 95\% CI, 1.06-2.23). Compared with weight maintenance, men who lost more than 11 pounds between 1982 and 1992 were at a decreased risk of nonmetastatic, high-grade prostate cancer (RR, 0.58; 95\% CI, 0.42-0.79) [58]. The relationship between prostate cancer and diabetes is more complex. Data from most epidemiological studies, such as the Northern Sweden Health and Disease Cohort, show that the risk was nonsignificantly inversely related to glucose levels. A modest decrease in prostate cancer risk has been consistently observed in men with diabetes. ${ }^{30,59}$

\section{Metabolic syndrome and breast cancer}

The incidence of breast cancer, type 2 diabetes, and metabolic syndrome has increased over the past few decades as a result of the obesity epidemic in both industrialized and developing countries. In a systematic review of epidemiologic studies of diabetes and the risk of breast cancer, Xue et al. noted a modest link between type 2 diabetes and the incidence of breast cancer. A meta-analysis of all available studies indicates that women with a history of diabetes have an approximately 16\% higher risk of developing breast cancer than do nondiabetic women, and this risk was most pronounced among postmenopausal women and those with type 2 diabetes. ${ }^{60}$ There has also been abundant data showing that various components of metabolic syndrome and insulin resistance are associated with elevated risk of postmenopausal breast cancer, including fasting insulin levels, abdominal obesity, and lipid profile. ${ }^{61}$ The biochemical mechanisms include extraglandular estrogen production, reduced sex hormone-binding globulin with consequent elevation of the bioactive plasma free estradiol, and increased insulin biosynthesis, all of which exert mitogenic effects on both untransformed and neoplastic breast epithelial cells. ${ }^{62}$ Current evidence supports the idea that a healthy lifestyle, including maintaining a healthful body weight, regular physical activity, and a healthy diet, can counter the growing epidemic of obesity, type 2 diabetes, and possibly breast cancer. Future studies are warranted to clarify the mechanism(s) responsible for the associations among type 2 diabetes, metabolic syndrome, and breast cancer and to develop effective intervention programs to prevent diabetes and breast cancer by promoting a healthy lifestyle. Data have confirmed the presence of metabolic syndrome as marker of poor prognosis in patients with breast cancer. In Italy, in 2006, one group tested the prognostic value of metabolic syndrome in 110 postmenopausal breast cancer patients, who participated in a 1-year dietary intervention study. The risk of adverse events after 5.5 years of follow up was examined using Cox's proportional hazard modeling, adjusting for hormone receptor status, stage at diagnosis, and serum testosterone level, all of which were shown to affect prognosis significantly. The adjusted HR for recurrence in the presence of metabolic syndrome at baseline was $3.0\left(95 \%\right.$ CI, 1.2-7.1). ${ }^{63}$ High BMI is associated inversely with risk of premenopausal breast cancer but positively with postmenopausal cancer in most available data. ${ }^{21,64}$

\section{Pathophysiology}

\section{Peroxisome proliferator-activated receptors and cancer}

Peroxisome proliferator-activated receptors (PPARs) are ligand-activated transcription factors that belong to the nuclear hormone receptor superfamily. These PPARs comprise three subtypes, PPAR $\alpha$, PPAR (also known as PPAR $\beta$ ), and PPAR $\gamma$, with a high degree of sequence conservation of each subtype across various species characterized. PPARs are linked to metabolic disorders and are interesting pharmaceutical targets. Recent evidence suggests that PPAR modulators may have beneficial effects as chemopreventive agents. ${ }^{65-67}$ PPAR $\alpha$, mainly expressed in the liver, activates fatty acid catabolism and has been used to treat dyslipidemia to lower plasma TG concentration. PPAR $\delta$ is expressed ubiquitously and is implicated in fatty acid oxidation and keratinocyte differentiation. PPAR $\delta$ agonists might form effective drugs for obesity, diabetes, and cardiovascular disease. PPAR $\gamma$ is involved in glucose metabolism through the improvement of insulin sensitivity and represents a potential therapeutic target of type 2 diabetes. PPAR $\gamma 2$ is expressed exclusively in adipose tissue and plays an important role in adipocyte differentiation. Thus, PPARs are molecular targets for the development of drugs treating metabolic syndrome. PPARs also play a role in the regulation of cancer cell growth (Table 2). The majority of studies in this field implicate PPAR $\gamma$ activation as an antitumorigenic and prodifferentiation factor, in contrast to the protumorigenic and less differentiated phenotype resulting from PPAR $\delta$ activation. ${ }^{66}$ There is emerging evidence that the nuclear receptor PPAR $y$ interacts with transcriptional factors to modulate cytokine production and action in immunity, inflammation, autoimmune diseases, and tumors. PPAR $\gamma$ regulation may occur at the levels of gene expression of cytokines themselves and their receptors or cytokine-mediated signaling transduction pathways in immune cells and cancer.

The crosstalk between PPARs and cytokine signaling pathways mediating inflammatory effects at the cellular level is also effective for inducing the expression of PPAR genes. The molecular basis of this interaction has remained elusive, despite the proposal of several distinct mechanisms. One of the most important mechanistic aspects is protein-protein interaction through a direct or cofactor-mediated indirect manner. On the basis of insights into the mechanisms on interaction between these two distinct families of transcriptional factors activated by different signaling pathways, new targeting drug design and/or therapeutic strategies will be 


\section{Table 2. Cancers That Have Evidence of PPAR- $\gamma$ EXPRESSION}

Skin-related cancers
Melanoma
Squamous cell carcinoma
Liposarcoma
Endocrine cancers
Pituitary tumor
Thyroid cancer
Adrenocortical carcinoma
Gastrointestinal cancers
Colonic tumor
Esophageal tumor
Gastric cancer
Pancreatic cancer
Hepatocellular carcinoma
Genito-urinary cancers
Testicular cancer
Breast tumor
Cervical carcinoma
Prostate cancer
Bladder cancer
Renal cell carcinoma
Other cancer
Lung tumor
Neuroblastoma

Abbreviation: PPAR- $\gamma$, Peroxisome proliferator-activated receptor- $\gamma$.

discovered and developed for treatment of cytokine-related diseases ranging from inflammation to cancer. ${ }^{68}$

\section{Insulin resistance}

Hyperinsulinemia or insulin resistence is believed to be the primary mechanism responsible for most of the manifestations of metabolic syndrome. Insulin is a major anabolic hormone that can stimulate cell proliferation. Adiposity induces adverse local and systemic effects that include adipocyte intracellular lipid accumulation, endoplasmic reticulum and mitochondrial stress, and insulin resistance, with associated changes in circulating adipokines, free fatty acids, and inflammatory mediators. Excess caloric intake and inadequate physical activity lead to an obesity epidemic and its related health consequences. In a study of insulin and fasting glucose and risk of recurrent colorectal adenomas, Flood et al. noted that patients with increased insulin and glucose are at higher risk of adenoma recurrence, and, for those with increased glucose, the increase in risk for recurrence of advanced adenomas is even greater. ${ }^{69}$

Because there have been abundant data showing overexpression of IGF-1 receptors on multiple human cancers, it is believed that the effects of insulin on cancer cell proliferation in vivo may involve an indirect mechanism, such as IGF-1 stimulation. Growth hormone is the primary stimulus for IGF-1 production in the liver and insulin can stimulate IGF-1 production by up-regulating growth hormone receptors in the liver. Hyperinsulinemia can also increase IGF-1 bioavailability by decreasing hepatic secretion of IGF-binding protein (IGFBP)-1 and $-2 .{ }^{44}$ IGF-1 has important proliferative and antiapoptotic effects in tumorigenesis. Angiogenesis is also stimulated by IGF-1 because it increases vascular endothelial growth factor (VEGF) production, which has been shown in breast and colon cancer cell lines. Activation of the IGF-1 receptor also stimulates the p21 ras/MAPK pathway for cell proliferation and the PI3K/AKT cell survival pathway. ${ }^{70,71}$ Hyperinsulinemia and IGF-1 are also believed to inhibit the synthesis of the sex hormone-binding globulin (SHBG), increasing levels of free sex hormones and promoting sex hormone-dependent cancers such as breast, endometrial, and prostate cancers. ${ }^{72}$ Also increasing evidence exists now that castration therapies used in prostate cancer may lead to hyperinsulinemia and raises concern for potential recurrence risk. ${ }^{73}$ Thus, hyperinsulinemia has been linked to neoplastic proliferation of various organ cells (Fig. 1).

Various population-based studies are available in humans showing a link to insulin resistance or hyperinsulinemia with various epithelial cancers. Initial studies done in prostate cancer showed a correlation with plasma IGF-1 levels. ${ }^{74}$ Subsequently, various studies have confirmed high levels of IGF-1 and insulin levels associated with prostate cancer risk prospectively. ${ }^{75-77} \mathrm{~A}$ link between breast cancer risk and hyperinsulinemia (measured by fasting C-peptide levels) has been shown mainly in postmenopausal breast cancer. ${ }^{78}$ High insulin levels have also been shown to be associated with risk of endometrial cancer independent of estradiol. ${ }^{79}$

\section{Cytokines and prostaglandins}

Cyclooxygenase and cancers. Cyclooxygenase-2 (COX-2) is an inducible enzyme produced by many cell types in response to multiple stimuli. Recently, COX-2 overexpression has been found in several types of human cancers, such as colon, breast, prostate, and pancreas, and appears to control many cellular processes. The contribution of COX-2 to carcinogenesis and the malignant phenotype of tumor cells have been thought to be related to its abilities to: (1) increase production of prostaglandins, (2) convert procarcinogens to carcinogens, (3) inhibit apoptosis, (4) promote angiogenesis, (5) modulate inflammation and immune function, and (6) increase tumor cell invasiveness. ${ }^{80}$ Among many solid tumors, pancreatic cancer has the worst prognosis, and inflammation has been identified as a significant factor in the development of pancreatic malignancy.

COX-2 and colon cancer. Although COX-2, the inducible isoform, is regularly expressed at low levels in colonic mucosa, its activity increases dramatically following mutation of the adenomatous polyposis coli (APC) gene, suggesting that $\beta$-catenin/T cell factor-mediated Wnt signaling activity may regulate COX-2 gene expression. In addition, hypoxic conditions and sodium butyrate exposure may also contribute to COX-2 gene transcription in human cancers. ${ }^{81}$ Because of its role in carcinogenesis, apoptosis, and angiogenesis, it is an excellent target for developing new drugs with selectivity for prevention and/or treatment of human cancers. ${ }^{82}$

Several cytokines, reactive oxygen species (ROS), and mediators of the inflammatory pathway, such as activation of nuclear factor- $\kappa \mathrm{B}(\mathrm{NF}-\kappa \mathrm{B})$ and COX-2, lead to an increase in cell proliferation, survival, and inhibition of the proapoptotic pathway, ultimately resulting in tumor angiogenesis, invasion, and metastasis. 


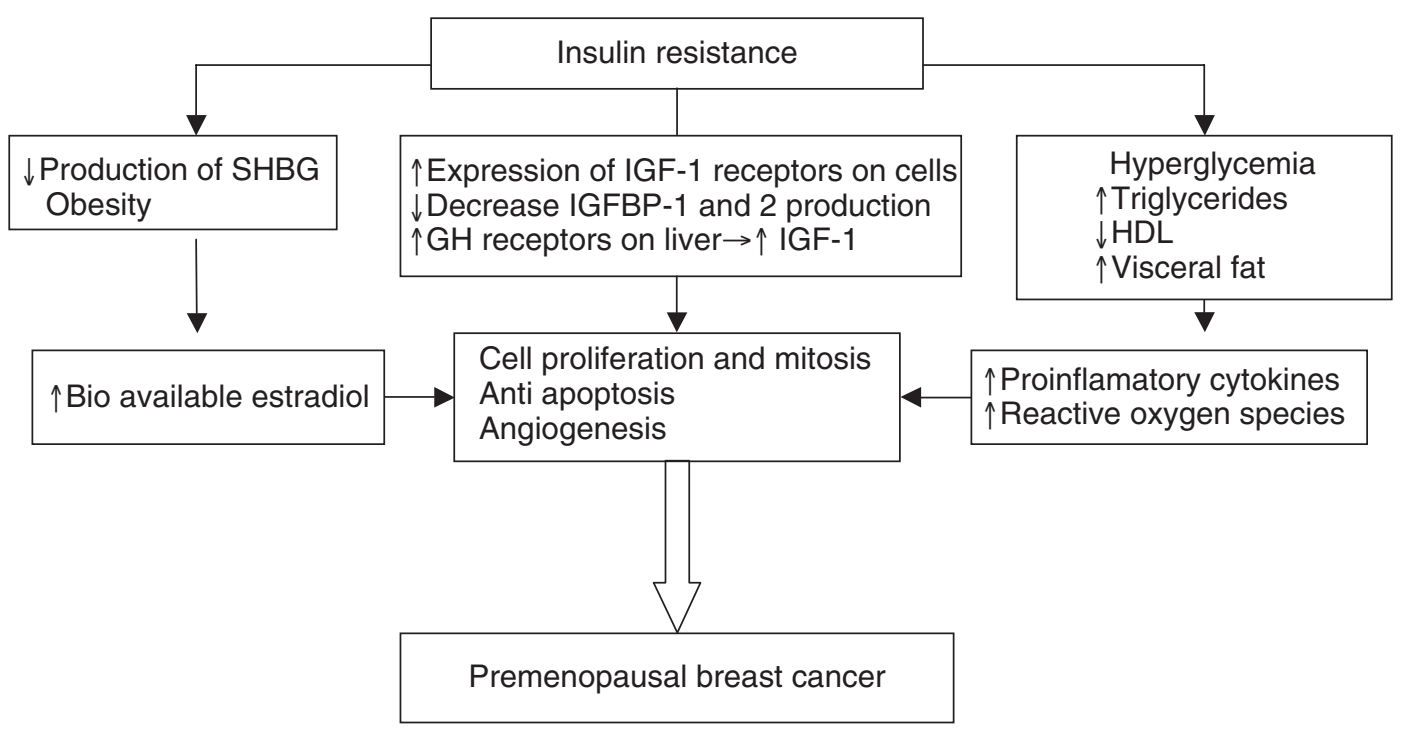

FIG. 1. Insulin resistance and premenopausal breast cancer. Abbreviations: SHBG, sex hormone-binding globulin; IGF, insulin-like growth factor; GH, growth hormone; HDL, high-density lipoprotein cholesterol.

\section{Proinflammatory cytokines}

Obesity is associated with both hypertrophy of preexisting adipocytes and hyperplasia due to the formation of new adipocytes. These clusters of adipocytes become hypoxic and secrete cytokines to stimulate angiogenesis into the adipose tissue. These adipocytes secrete a number of proinflammatory cytokines such as tumor necrosis factor$\alpha \mathrm{TNF}-\alpha$, interleukin-6 (IL-6), IL-8, IL-10, macrophage inflammatory protein 1 (MIP-1), and monocyte chemoattractant protein-1 (MCP-1). These cytokines secreted by adipocytes are known to promote insulin resistance and increase circulating TG, features of the metabolic syndrome.

Inflammation has also been linked to many types of cancer, such as gastric, pancreatic, esophageal, liver, bladder, and colorectal cancers, because it influences growth, apoptosis, and proliferation of tumor and stromal cells. Increased circulating cytokines from adipocytes promote cancer progression by contributing to inflammation and ROS formation. Proinflammatory cytokines implicated in carcinogenesis include IL-1, IL-6, IL-15, colony-stimulating factors, TNF- $\alpha$, and the macrophage migration inhibitory factor. IL-6 is the key growth and survival factor of multiple myeloma (MM) cells, and is particularly involved in the origin of all benign and malignant plasma cell expansions as well as MM cell resistance. Humoral cytokines promote metastasis in hepatocellular carcinoma. Likewise, a signature consisting of 11 cytokine genes in the lung environment predicted lymph node metastasis and prognosis of lung adenocarcinoma, with IL- 8 and TNF- $\alpha$ as the top two genes for predicting prognosis. IL-8 can have angiogenic activities in several cancers, including non-small cell lung cancer, and can function as a positive autocrine growth factor. Both TNF- $\alpha$ and IL-6 contributed to chemically induced skin tumors and lymphomas in mice.

Collectively, cytokines are considered as a linker between inflammation and cancer. Cytokines, ROS, and mediators of the inflammatory pathway (e.g., NF-кB and COX-2) have been shown to increase cell cycling, cause loss of tumor suppressor function, and stimulate oncogene expression and lead to cancers. Positive feedback mechanisms between estrogens and inflammatory factors may exist in the breast and contribute to hormone-dependent breast cancer growth and progression. ${ }^{83}$ Prostaglandin E synthase (PTGES) is also up-regulated by the proinflammatory cytokines TNF- $\alpha$ or IL-1 $\beta$. Cytokines can enhance estrogen receptor (ER) activity and PTGES expression through the NF-кB pathway and cytokines can act to up-regulate aromatase expression as well as $17 \beta$-hydroxysteroid dehydrogenase activity in breast tissue, thereby leading to a further increase in E2 production. ${ }^{83}$ Because insulin resistance is directly related to the metabolic syndrome and cancer development, adipokines may play a crucial role in linking these two diseases.

\section{Role of adiponectin}

Adiponectin, which is also referred to as ACP30 (Acrp30), is secreted predominantly by white adipose tissue. ${ }^{84}$ Circulating concentrations of adiponectin are reduced in obesity and type 2 diabetes and have a strong inverse association with central obesity, independent of age, menopausal status, and estradiol concentrations. ${ }^{85}$ Adiponectin is considered to have beneficial antineoplastic effects, which are believed to be due to antiproliferative, antiinflamatory effects, along with antagonizing insulin resistance. ${ }^{86}$ In a recent study, adiponectin was shown to act on preneoplastic colon epithelial cells to regulate cell growth via two distinct pathways inhibiting leptin-induced NF-кB-dependent autocrine IL- 6 production and trans-IL- 6 signaling in a model of preneoplastic (IMCE) colon epithelial cells. ${ }^{87}$ In a number of epidemiological studies, adiponectin levels have been linked to breast cancer and are believed to inhibit breast cancer cell proliferation in vivo. This effect may be due to adiponectin-triggered cellular apoptosis in MDA-MB-231 breast cancer cells in the presence of $17 \beta$-estradiol. These findings may suggest that a cross-talk between adiponectin 
and estrogen receptor signaling exists in breast cancer cells and that adiponectin effects on the growth and apoptosis of breast cancer cells in vitro are dependent on the presence of $17 \beta$-estradiol. ${ }^{88}$ Circulating adiponectin concentrations are inversely correlated with the incidence of endometrial carcinoma in epidemiological studies. A recent study investigated the direct effects of adiponectin on two endometrial carcinoma cell lines, HEC-1-A and RL95-2. Adiponectin treatment led to suppression of cell proliferation in both cell types, which was primarily believed to be due to the significant increase of cell populations at $\mathrm{G}_{1} / \mathrm{G}_{0}$ phase and secondary to the induction of apoptosis. ${ }^{89}$

Data on adiponectin levels in prostate cancer are more conflicting. Adiponectin may act as a novel inhibitor in prostate cancer cell growth.${ }^{90}$ More published data show that adipokines may contribute to the molecular basis for the association between obesity and prostate cancer. However, data from a small case-control study performed in Turkey by Goktas et al. ${ }^{91}$ showed a relationship between hypoadiponectinemia and carcinoma of the prostate. Plasma adiponectin levels were significantly lower in a group of 30 prostate cancer patients than in 41 patients with benign prostatic hyperplasia and 36 healthy controls. Furthermore, the adiponectin concentrations were inversely correlated with the tumor grade and disease stage. An important feature of this study was that obese males were excluded from the study and that BMI was not statistically different among all three groups. However, further prospective studies have failed to show this association..$^{2}$ Epidemilogical data have also shown a link between adiponectin levels and renal cell cancer, ${ }^{93}$ leukemia, ${ }^{94}$ and certain lymphomas. ${ }^{95}$

\section{Therapeutic Considerations}

Therapeutic considerations need to focus on reduction of the risk factors. Various therapeutic interventions for weight reduction and healthy life style have been linked to a reduced cancer risk in the general population. A recently published study assessing mortality after bariatric surgery showed a significant decrease in mortality from cancerrelated deaths in the bariatric surgery group compared both with all subjects and matched subjects. The HRs were 0.38 and 0.40 , respectively, which were statistically significant values $(P<0.001){ }^{96}$

Metformin is the insulin sensitizer used primarily in the treatment of type 2 diabetes mellitus. When compared with other insulin secretogauges like sulfonylurea or insulin, it appears to confer a cancer-protective effect in diabetic individuals believed to be secondary to decreasing insulin resistance. ${ }^{97}$ This was validated from the data from a population-based cohort in Canada, reported in Diabetes Care, which showed that patients with type 2 diabetes exposed to sulfonylureas and exogenous insulin had a significantly increased risk of cancer-related mortality compared with patients exposed to metformin. ${ }^{98}$

Anticytokine vaccines, inhibitors of proinflammatory NF- $\kappa \mathrm{B}$ and COX-2 pathways, thiazolidinediones, and antioxidants are potentially useful for the prevention or treatment of pancreatic cancer. Similarly epidemiologic studies have documented a $40-50 \%$ reduction in the incidence of colorectal cancer in individuals taking nonsteroidal antiinflammatory drugs (NSAIDs). The long-term use of COX2-selective inhibitors has, unfortunately, demonstrated cardiovascular toxicity, so their use in cancer prevention and therapy is currently questionable. However, there is evidence suggesting that further development of novel COX-2-selective agents is needed for the prevention and/or treatment of human cancers, especially pancreatic cancer. PPARs are molecular targets for the development of drugs treating metabolic syndrome. However, PPARs also play a role in the regulation of cancer cell growth. Recent evidence suggests that PPAR modulators may have beneficial effects as chemopreventive agents. ${ }^{67}$

\section{Conclusions}

Available evidence from epidemiological, clinical, translational, and experimental studies supports the hypothesis that metabolic syndrome or components of metabolic syndrome may be important etiologic factors for certain cancers, progression of some cancers as well as altering the outcome of some cancers. While research continues to work toward preventing cancers, nutritional and lifestyle modifications may alleviate metabolic syndrome and reduce the risk.

\section{References}

1. Reaven GM. Banting lecture 1988 Role of insulin resistance in human disease. Diabetes 1988;37:1595-1607.

2. Lakka HM et al. The metabolic syndrome and total and cardiovascular disease mortality in middle-aged men. JAMA 2002;288:2709-2716

3. Alberti KG. Zimmet P, Shaw J. Metabolic syndrome-a new world-wide definition. A Consensus Statement from the International Diabetes Federation. Diabet Med 2006;23:469-480.

4. Ford ES. Insulin resistance syndrome: the public health challenge. Endocr Pract 2003;9(Suppl 2):23-25.

5. Despres JP et al. Hyperinsulinemia as an independent risk factor for ischemic heart disease. N Engl J Med 1996;334:952.

6. Pyorala $\mathrm{M}$ et al. Hyperinsulinemia predicts coronary heart disease risk in healthy middle-aged men: the 22-year followup results of the Helsinki Policemen Study. Circulation 1998; 98:398-404.

7. Third Report of the National Cholesterol Education Program (NCEP) Expert Panel on Detection Evaluation and Treatment of High Blood Cholesterol in Adults (Adult Treatment Panel III) final report. Circulation 2002 106:3143-1421.

8. Ford ES Giles WH, Dietz WH. Prevalence of the metabolic syndrome among US adults: findings from the third National Health and Nutrition Examination Survey. JAMA 2002 287:356-359.

9. Russo A, Autelitano M, Bisanti L. Metabolic syndrome and cancer risk. Eur J Cancer 2008 44:293-297.

10. Larsson SC, Wolk A. Obesity and colon and rectal cancer risk: a meta-analysis of prospective studies. Am J Clin Nutr 2007;86:556-565.

11. Larsson SC, Wolk A. Overweight obesity and risk of liver cancer: a meta-analysis of cohort studies. Br J Cancer 2007;97:1005-1008.

12. Larsson SC, Wolk A. Body mass index and risk of multiple myeloma: a meta-analysis. Int J Cancer 2007;121:2512-2516.

13. Larsson SC, Wolk A. Obesity and risk of non-Hodgkin's lymphoma: a meta-analysis. Int J Cancer 2007;121:1564-1570.

14. Larsson SC, Wolk A. Obesity and the risk of gallbladder cancer: a meta-analysis. Br J Cancer 2007;96:1457-1461.

15. Larsson SC, Orsini N, Wolk A. Body mass index and pancreatic cancer risk: A meta-analysis of prospective studies. Int J Cancer 2007;120:1993-1998.

16. Larsson SC, Wolk A. Overweight and obesity and incidence of leukemia: a meta-analysis of cohort studies. Int J Cancer 2008;122:1418-1421. 
17. Olsen $\mathrm{CM}$ et al. Body size and risk of epithelial ovarian and related cancers: a population-based case-control study. Int $J$ Cancer 2008;123:450-456.

18. Jensen A et al. Risk of breast cancer and gynecologic cancers in a large population of nearly 50000 infertile Danish women. Am J Epidemiol 2008;168:49-57.

19. McCourt CK et al. Body mass index: relationship to clinical pathologic and features of microsatellite instability in endometrial cancer. Gynecol Oncol 2007;104:535-539.

20. Patel AV et al. The role of body weight in the relationship between physical activity and endometrial cancer: Results from a large cohort of US women. Int J Cancer 2008;123:1877-1882.

21. Reeves GK et al. Cancer incidence and mortality in relation to body mass index in the Million Women Study: cohort study. BMJ 2007;335:1134.

22. Gago-Dominguez M et al. Lipid peroxidation: a novel and unifying concept of the etiology of renal cell carcinoma (United States). Cancer Causes Control 2002;13:287-293.

23. Gago-Dominguez M, Jiang X, Castelao JE. Lipid peroxidation oxidative stress genes and dietary factors in breast cancer protection: a hypothesis. Breast Cancer Res 2007;9:201.

24. Carmichael AR. Obesity and prognosis of breast cancer. Obes Rev 2006;7:333-340.

25. Dignam JJ et al. Body mass index and outcomes in patients who receive adjuvant chemotherapy for colon cancer. J Natl Cancer Inst 2006;98:1647-1654.

26. Haydon AM et al. Effect of physical activity and body size on survival after diagnosis with colorectal cancer. Gut 2006; 55:62-67.

27. Levine $\mathrm{W}$ et al. Post-load plasma glucose and cancer mortality in middle-aged men and women 12-year follow-up findings of the Chicago Heart Association Detection Project in Industry. Am J Epidemiol 1990;131:254-262.

28. Jee $\mathrm{SH}$ et al. Fasting serum glucose level and cancer risk in Korean men and women. JAMA 2005;293:194-202.

29. Rapp $K$ et al. Fasting blood glucose and cancer risk in a cohort of more than 140000 adults in Austria. Diabetologia 2006;49:945-952.

30. Stattin P et al. Prospective study of hyperglycemia and cancer risk. Diabetes Care 2007;30:561-567.

31. Frayling TM, Colhoun H, Florez JC. A genetic link between type 2 diabetes and prostate cancer. Diabetologia 2008;51:1757-60.

32. Berster JM, Goke B. Type 2 diabetes mellitus as risk factor for colorectal cancer. Arch Physiol Biochem 2008;114:84-98.

33. Yang YX, Hennessy S, Lewis JD. Type 2 diabetes mellitus and the risk of colorectal cancer. Clin Gastroenterol Hepatol 2005;3:587-594.

34. Chung YW et al. Insulin therapy and colorectal adenoma risk among patients with Type 2 diabetes mellitus: a case-control study in Korea. Dis Colon Rectum 2008;51:593-597.

35. Yang YX, Hennessy S, Lewis JD. Insulin therapy and colorectal cancer risk among type 2 diabetes mellitus patients. Gastroenterology 2004;127:1044-1050.

36. Kucharska-Newton AM et al. HDL-cholesterol and the incidence of lung cancer in the Atherosclerosis Risk in Communities (ARIC) study. Lung Cancer 2008;61:292-300.

37. Shor $\mathrm{R}$ et al. Low HDL levels and the risk of death sepsis and malignancy. Clin Res Cardiol 2008;97:227-233.

38. Shor R et al. Low serum LDL cholesterol levels and the risk of fever sepsis and malignancy. Ann Clin Lab Sci 2007;37:343-348.

39. Gaard M, Tretli S, Urdal P. Risk of breast cancer in relation to blood lipids: a prospective study of 31209 Norwegian women. Cancer Causes Control 1994;5:501-509.

40. Wuermli L et al. Hypertriglyceridemia as a possible risk factor for prostate cancer. Prostate Cancer Prostatic Dis 2005;8:316-320.

41. Bowers $\mathrm{K}$ et al. A prospective study of anthropometric and clinical measurements associated with insulin resistance syndrome and colorectal cancer in male smokers. Am J Epidemiol 2006;164:652-664.
42. Chiu HM et al. Association of metabolic syndrome with proximal and synchronous colorectal neoplasm. Clin Gastroenterol Hepatol 2007;5:221-229; quiz 141.

43. Colangelo LA et al. Colorectal cancer mortality and factors related to the insulin resistance syndrome. Cancer Epidemiol Biomarkers Prev 2002;11:385-391.

44. Cowey S, Hardy RW. The metabolic syndrome. A high-risk state for cancer? Am J Pathol 2006;169:1505-1522.

45. Giovannucci E. Metabolic syndrome hyperinsulinemia and colon cancer: a review. Am J Clin Nutr 2007;86:s836-s842.

46. $\mathrm{Kim} \mathrm{JH}$ et al. Is metabolic syndrome a risk factor for colorectal adenoma? Cancer Epidemiol Biomarkers Prev 2007;16:1543-1546.

47. Morita $\mathrm{T}$ et al. The metabolic syndrome is associated with increased risk of colorectal adenoma development: the SelfDefense Forces health study. Asian Pac J Cancer Prev 2005; 6:485-489.

48. Pischon T et al. Body size and risk of colon and rectal cancer in the European Prospective Investigation Into Cancer and Nutrition (EPIC). J Natl Cancer Inst 2006;98:920-931.

49. Sturmer $\mathrm{T}$ et al. Metabolic abnormalities and risk for colorectal cancer in the physicians' health study. Cancer Epidemiol Biomarkers Prev 2006;15:2391-2397.

50. Hu FB et al. Prospective study of adult onset diabetes mellitus (type 2) and risk of colorectal cancer in women. J Natl Cancer Inst 1999;91:542-547.

51. Limburg PJ et al. Diabetes mellitus and subsite-specific colorectal cancer risks in the Iowa Women's Health Study. Cancer Epidemiol Biomarkers Prev 2005;14:133-137.

52. Tran TT et al. Hyperinsulinemia but not other factors associated with insulin resistance acutely enhances colorectal epithelial proliferation in vivo. Endocrinology 2006;147:1830-1837.

53. Giovannucci E. Insulin insulin-like growth factors and colon cancer: a review of the evidence. J Nutr 2001;131(11 Suppl):3109S-3120S.

54. Engeland A Tretli S, Bjorge T. Height body mass index and prostate cancer: a follow-up of 950000 Norwegian men. Br J Cancer 2003:89:1237-1242.

55. Wolk A et al. A prospective study of obesity and cancer risk (Sweden). Cancer Causes Control 2001;12:13-21.

56. MacInnis RJ, English DR. Body size and composition and prostate cancer risk: systematic review and meta-regression analysis. Cancer Causes Control 2006;17:989-1003.

57. Robinson WR et al. Obesity before age 30 years and risk of advanced prostate cancer. Am J Epidemiol 2005;161:1107-1114.

58. Rodriguez $\mathrm{C}$ et al. Body mass index weight change and risk of prostate cancer in the Cancer Prevention Study II Nutrition Cohort. Cancer Epidemiol Biomarkers Prev 2007;16:63-69.

59. Bonovas S, Filioussi K, Tsantes A. Diabetes mellitus and risk of prostate cancer: a meta-analysis. Diabetologia 2004;47:1071-1078.

60. Xue F, Michels KB. Diabetes metabolic syndrome and breast cancer: a review of the current evidence. Am J Clin Nutr 2007;86:s823-s835.

61. Lawlor DA, Smith GD, Ebrahim S. Hyperinsulinaemia and increased risk of breast cancer: findings from the British Women's Heart and Health Study. Cancer Causes Control 2004;15:267-275.

62. Vona-Davis L, Howard-McNatt M, Rose DP. Adiposity type 2 diabetes and the metabolic syndrome in breast cancer. Obes Rev 2007;8:395-408.

63. Pasanisi $\mathrm{P}$ et al. Metabolic syndrome as a prognostic factor for breast cancer recurrences. Int J Cancer 2006;119:236-238.

64. Ursin G et al. A meta-analysis of body mass index and risk of premenopausal breast cancer. Epidemiology 1995;6:137-141.

65. Glazer RI et al. PPARgamma and PPARdelta as modulators of neoplasia and cell fate. PPAR Res 2008:247379.

66. Sertznig $P$ et al. Present concepts and future outlook: function of peroxisome proliferator-activated receptors (PPARs) for pathogenesis progression and therapy of cancer. J Cell Physiol 2007;212:1-12. 
67. Tachibana $\mathrm{K}$ et al. The role of PPARs in cancer. PPAR Res 2008:102737.

68. Yang XY, Wang LH, Farrar WL. A role for PPARgamma in the regulation of cytokines in immune cells and cancer. PPAR Res 2008:961753.

69. Flood A et al. Elevated serum concentrations of insulin and glucose increase risk of recurrent colorectal adenomas. Gastroenterology 2007;133:1423-1429.

70. Hoeben A et al. Vascular endothelial growth factor and angiogenesis. Pharmacol Rev 2004;56:549-580.

71. Ibrahim YH, Yee D. Insulin-like growth factor-I and cancer risk. Growth Horm IGF Res 2004;14:261-269.

72. Calle EE, Kaaks R. Overweight obesity and cancer: epidemiological evidence and proposed mechanisms. Nat Rev Cancer 2004;4:579-591.

73. Smith MR, Lee $H$, Nathan DM. Insulin sensitivity during combined androgen blockade for prostate cancer. J Clin Endocrinol Metab 2006;91:1305-1308.

74. Chan JM et al. Plasma insulin-like growth factor-I and prostate cancer risk: a prospective study. Science 1998;279:563-566.

75. Giovannucci E et al. Height predictors of C-peptide and cancer risk in men. Int J Epidemiol 2004;33:217-225.

76. Harman SM et al. Serum levels of insulin-like growth factor I (IGF-I) IGF-II IGF-binding protein-3 and prostate-specific antigen as predictors of clinical prostate cancer. J Clin Endocrinol Metab 2000;85:4258-4265.

77. Kaaks $\mathrm{R}$ et al. Interrelationships between plasma testosterone SHBG IGF-I insulin and leptin in prostate cancer cases and controls. Eur J Cancer Prev 2003;12:309-315.

78. Verheus $M$ et al. Serum C-peptide levels and breast cancer risk: results from the European Prospective Investigation into Cancer and Nutrition (EPIC). Int J Cancer 2006;119:659-667.

79. Gunter MJ et al. A prospective evaluation of insulin and insulin-like growth factor-I as risk factors for endometrial cancer. Cancer Epidemiol Biomarkers Prev 2008;17:921-929.

80. Xu XC. COX-2 inhibitors in cancer treatment and prevention a recent development. Anticancer Drugs 2002;13:127-137.

81. Dempke W et al. Cyclooxygenase-2: a novel target for cancer chemotherapy? J Cancer Res Clin Oncol 2001;127:411-417.

82. Sarkar FH et al. Back to the future: COX-2 inhibitors for chemoprevention and cancer therapy. Mini Rev Med Chem 2007;7:599-608

83. Frasor J et al. Synergistic up-regulation of prostaglandin E synthase expression in breast cancer cells by $17 \beta$-estradiol and proinflammatory cytokines. Endocrinology 2008;149:6272-6279.

84. Scherer PE et al. A novel serum protein similar to C1q produced exclusively in adipocytes. J Biol Chem 1995;270 26746-26749.

85. Gavrila A et al. Serum adiponectin levels are inversely associated with overall and central fat distribution but are not directly regulated by acute fasting or leptin administration in humans: cross-sectional and interventional studies. J Clin Endocrinol Metab 2003;88:4823-4831.

86. Barb D et al. Adiponectin in relation to malignancies: a review of existing basic research and clinical evidence. Am J Clin Nutr 2007;86:s858-s866.

87. Fenton JI et al. Adiponectin blocks multiple signaling cascades associated with leptin-induced cell proliferation in Apc Min/+ colon epithelial cells. Int J Cancer 2008;122:2437-2445.

88. Pfeiler GH et al. Adiponectin effects on human breast cancer cells are dependent on 17-beta estradiol. Oncol Rep 2008;19:787-793.

89. Cong L et al. Human adiponectin inhibits cell growth and induces apoptosis in human endometrial carcinoma cells HEC1-A and RL95 2. Endocr Relat Cancer 2007;14:713-720.

90. Bub JD, Miyazaki T, Iwamoto Y. Adiponectin as a growth inhibitor in prostate cancer cells. Biochem Biophys Res Commun 2006;340:1158-1166.

91. Goktas S et al. Prostate cancer and adiponectin. Urology 2005;65:1168-1172.

92. Baillargeon $\mathrm{J}$ et al. Obesity adipokines and prostate cancer in a prospective population-based study. Cancer Epidemiol Biomarkers Prev 2006;15:1331-1335.

93. Pamuk GE et al. Increased adiponectin level in non-Hodgkin lymphoma and its relationship with interleukin-10 Correlation with clinical features and outcome. J Exp Clin Cancer Res 2006;25:537-541.

94. Spyridopoulos TN et al. Low adiponectin levels are associated with renal cell carcinoma: a case-control study. Int J Cancer 2007;120:1573-1578.

95. Avcu $\mathrm{F}$ et al. Association of plasma adiponectin concentrations with chronic lymphocytic leukemia and myeloproliferative diseases. Int J Hematol 2006;83:254-258.

96. Adams TD et al. Long-term mortality after gastric bypass surgery. N Engl J Med 2007;357:753-761.

97. Bowker SL et al. Increased cancer-related mortality for patients with type 2 diabetes who use sulfonylureas or insulin. Diabetes Care 2006;29:254-258.

98. Evans JM et al. Metformin and reduced risk of cancer in diabetic patients BMJ 2005;330:1304-1305.

99. Alberti KG, Zimmet P, Shaw J. The metabolic syndrome-a new worldwide definition. Lancet 2005:366:1059-1062.

Address correspondence to: Subhashini Yaturu, M.D. Department of Endocrinology

Louisiana State University Health Sciences Center 510 East Stoner Ave, 111E Shreveport, LA 71101-4295

E-mail: Subhashini.Yaturu@va.gov; yaturu@yahoo.com 
